Revista Oficial de la Federación Iberoamericana de Asociaciones de Psicología (FIAP)

[Official Journal of the Latin-American Federation of Psychological Associations]

\title{
Efectos del consumo dual cannabis-tabaco sobre el embarazo y la descendencia: una revisión sistemática
}

\section{Francisco González-Sala ${ }^{1 *}$,Víctor Ciudad-Fernández', Julia Haba-Osca' y Julia Osca-Lluch ${ }^{2}$}

Universidad de Valencia

${ }^{2}$ Instituto de Gestión e Innovación del Conocimiento INGENIO

- Recibido: 29 - 12 - 2019 - Aceptado: 09 - 04 - 2020 . Avance Online: 17 - 04 - 2020

RESUMEN: El objetivo del presente estudio ha sido la realización de una revisión de los trabajos publicados que han estudiado el consumo conjunto cannabis/marihuana y tabaco en mujeres embarazadas y las posibles consecuencias sobre su descendencia. Se han utilizado como fuentes de información las bases de datos de la Web of Science, Scielo y Medline. Se recuperaron un total de 240 artículos, de los que solamente 16 cumplían los requisitos de inclusión en este estudio. Los resultados apuntan a un perfil psicosocial de riesgo en embarazadas con consumo dual cannabis-tabaco y efectos sobre el desarrollo fetal según diferentes parámetros, como circunferencia de la cabeza, peso y longitud al nacer, prematuridad, edad gestacional y defectos en el recién nacido, entre otros. Sin embargo no hay diferencias en las puntuaciones en el test de Apgar, hipertensión arterial, diabetes gestacional o medicalización del parto. El consumo conjunto cannabis-tabaco tiene un efecto aditivo con respecto a algunos indicadores del desarrollo fetal. Se requiere de intervenciones preventivas a diferentes niveles a la hora de abordar el consumo dual cannabis-tabaco en embarazadas, considerando éste un problema de salud pública.

PALABRAS CAVE: Consumo dual; Cannabis; Tabaco; Embarazo; Descendencia.

\section{Effects of dual cannabis-tobacco consumption on pregnancy and offspring: a systematic review}

ABSTRACT: The objective of the present study has been to carry out a review of the published works that have studied cannabis/marijuana and tobacco co-use in pregnant women and the possible consequences on their offspring The sources of information the databases used were the Web of Science (WoS), Scielo and Medline. A total of 240 articles were recovered, of which only 16 met the inclusion requirements in this study. The results point to a psychosocial profile of risk in pregnant women with dual consumption cannabis-tobacco and effects of co-use of these substances on fetal development according to different parameters, such as head circumference, weight and length at birth, prematurity, gestational age and defects in the newborn, among others. However, there are no differences in the scores in the Apgar test, hypertension, gestational diabetes or medicalization of the baby delivery. Cannabis-tobacco co-use has an additive effect with respect to some indicators of fetal development. Preventive interventions are required different levels when dealing with cannabis-tobacco co-use in pregnant women, considering this a major public health problem.

KEY WORDS: Co-use; Cannabis; Tobacco; Pregnancy; Offspring.

El consumo dual de cannabis y tabaco ha aumentado entre adolescentes y adultos de forma considerable (Ramo, Liv, y Prochaska, 2012). El consumo de cannabis va asociado

*Correspondence: Francisco González Sala.

Universidad de Valencia

CP: 46019, Valencia, España.

E-mail: Francisco.Gonzalez-Sala@uv.es

(c) 2020 Sociedad Universitaria de Investigación en Psicología y Salud. Publicado por Consejo General de Colegios Oficiales de Psicólogos, España. Este es un artículo Open Access
bajo la CC BY-NC-ND licencia (http://creativecommons.org/licencias/by-nc-nd/4.0/)

Citar como/Cite as: González-Sala, F., Ciudad-Fernández, V., Haba-Osca, J. y OscaLilach, J. (2020). Efectos del consumo dual cannabis-tabaco sobre el embarazo y la descendencia: una revisión sistemática. (Effects of dual cannabis-tobacco y la descendencia: una revision sistematica. (Effects of dual cannabis-tobacco
consumption on pregnancy and offspring: a systematic review). Revista lberoamericana de Psicología y Salud, 17 (2), 68-81. https://doi.org/10.23923/i.rips.2020.02.036 al consumo de tabaco, de tal forma que prácticamente más de tres cuartas partes de las personas que consumen cannabis lo hacen con tabaco (Richter et al., 2004; Schaver, Berg, Kegler, Donovan, y Windle, 2015; 2016). La relación entre el consumo conjunto de ambas sustancias es tan evidente que como apunta Ramo, Liu y Prochaska (2013), el uso de una sustancia o la otra lleva asociado el incremento de éstas, relación que también se observa entre 
mujeres gestantes (Fried, Watkinson, y Willian, 1984; Gray et al., 2010).

En el caso del consumo dual cannabistabaco en embarazadas, en Estados Unidos Coleman-Cowger, Schaver y Peters (2017) lo sitúan en un 3.3\% frente al $5.7 \%$ en mujeres no embarazadas. En otros estudios, ColemanCowger, Oga, Peters y Mark (2018) encuentran que un $9 \%$ de embarazadas son co-usuarias de ambas sustancias. Entre las embarazadas que consumen marihuana, entre un $43 \%$ y un $86 \%$ lo hace con tabaco (El Marroun et al., 2008; Chabarria et al., 2016; Gray et al., 2010; Mark, Desai, y Terplan, 2016; Moore et al., 2010; Oga, Mark, y Coleman-Cowger, 2017; SaurelCubizolles, Prunet, y Blondel, 2014). Brown et al. (2016) y Young-Wolff et al. (2017) apuntan a un aumento del consumo de cannabis y del consumo conjunto cannabis-tabaco en madres gestantes.

El Marroun et al. (2008), Emery et al. (2016), Ko, Farr, Tong, Greanga y Callaghan (2015) y Lester et al. (2001) afirman que entre las mujeres con un Trastorno de Dependencia al Cannabis y aquellas con consumo dual existe un mayor riesgo de seguir consumiendo durante el embarazo y el postparto. Para Passey, SansonFisher, D'Este y Stirling (2014) las mujeres con consumo compartido de cannabis, tabaco o alcohol tienen más probabilidades de usar una de estas sustancias en el embarazo.

Con respecto a la evolución del consumo a lo largo de la gestación, Gray et al. (2010) y Oga et al. (2018) apuntan a una disminución en el consumo. En el caso del consumo dual cannabis-tabaco los mayores consumos se dan en el primer trimestre, reduciéndose a medida que avanza el embarazo (Coleman-Cowger et al., 2017; Oga et al. 2018; Stroud et al. 2018), siendo el descenso mayor en el caso del alcohol pero no tan evidente en el consumo de tabaco o cannabis (Passey et al., 2014). Por su parte Moore et al. (2010) no encuentran una disminución en el consumo de tabaco y de cannabis a lo largo del embarazo.

El objetivo del presente estudio es realizar una revisión de los trabajos que han estudiado el consumo dual cannabis/marihuana y tabaco en mujeres embarazadas y las posibles consecuencias sobre la descendencia, ofreciendo datos sobre el perfil psicosocial de la mujer, las consecuencias sobre el embarazo y el desarrollo pre, peri y postnatal, con el fin de orientar las intervenciones en embarazadas consumidoras de ambas sustancias.

\section{METODO}

\section{- PROCEDIMIENTO Y MATERIALES}

La revisión sistemática efectuada se basó en la metodología PRISMA (Liberati et al., 2009). Se realizaron diferentes búsquedas bibliográficas relacionadas con el consumo dual cannabistabaco en las bases de datos de la Web of Science (a través de la colección principal), Scielo (a través de la Web of Science) y Medline (a través de la Web of Science). Las búsquedas se realizaron durante el mes de febrero de 2019. Los descriptores empleados fueron (cannabis or marijuana or marihuana) and (tobacco or smoking or smoker or cigarrete*) and (co-use or consumption) and (pregnan*). Se localizaron un total de 240 artículos científicos, de los cuales se eliminaron 19 por no estar escritos en inglés o castellano, 3 por estar repetidos y 1 trabajo por tratarse de un comentario de un artículo. Con los 217 artículos seleccionados se realizó una primera revisión del título y del abstract, con el fin de determinar si los trabajos abordaban el consumo conjunto cannabis-tabaco en embarazadas, seleccionándose un total de 35 trabajos. Tras su lectura completa se descartaron 19 por no incluir un grupo de consumo dual en el estudio, no abordar el perfil psicosocial de las gestantes con consumo dual o ser trabajos que hablaban únicamente sobre la prevalencia del consumo dual cannabis-tabaco. De esta forma, fueron 16 el número de artículos seleccionados (ver Figura 1).

\section{LOS CRITERIOS DE INCLUSIÓN FUERON:}

- Artículos científicos relacionados con el consumo conjunto cannabis-tabaco en embarazadas y el desarrollo pre, peri y postnatal desde diferentes ámbitos.

- Publicados entre 1900 y 2018 en castellano y en inglés. 
- Presencia de un grupo de consumo dual cannabis-tabaco.

\section{LOS CRITERIOS DE EXCLUSIÓN FUERON:}

- Abordar el consumo de cannabis o tabaco por separado.

- Estudios dirigidos al abandono del consumo dual después del embarazo.

- Otras tipologías documentales, actas de congreso, libros, capítulos de libros.

- Artículos que solo aportasen datos de prevalencia del consumo dual cannabis-tabaco.

- Artículos publicados en otros idiomas distintos del castellano y del inglés.

\section{-RIESGO DE SESGO: CALIDAD METODOLÓGICA}

La calidad metodológica de los estudios seleccionados para la presente revisión sistemática se realizó a través de 8 de los 18 indicadores que recoge la escala de calidad QUIRE Guidelines 2.0 (Ogrinc et al., 2015). Los indicadores empleados para la evaluación de la calidad fueron título, resumen, medias, análisis, resultados, limitaciones y conclusiones. En base a estos indicadores se procedió a clasificar los trabajos en cinco categorías según su calidad, baja, media baja, media, media alta y alta. Este proceso se realizó por dos investigadores valorando cada uno de los indicadores de calidad seleccionados, concluyendo que los artículos incluidos en la revisión se encontraban en una categoría alta, por lo que no se procedió a la eliminación de ninguno de los artículos seleccionados.

\section{-FIABILIDAD DE LA CODIFICACIÓN.}

El proceso de búsqueda y cribaje de los registros se realizó por dos investigadores expertos. Tras la selección de los 16 trabajos incluidos en la revisión sistemática, estos fueron codificados según las variables incluidas en la revisión por dos investigadores con experiencia, repartiéndose 8 trabajos cada uno de ellos. Este proceso se realizó en dos pasos. En primer lugar, tras una primera codificación (momento 1), se volvieron a codificar las variables por el mismo investigador en menos de una semana (momento 2). En un segundo paso se les pidió que codificaran los artículos que había codificado el otro investigador. Todo ello con el objetivo de comprobar el acuerdo intercodificación e intra-codificación. La evaluación de la codificación se realizó mediante la técnica de análisis de contenido categorial aplicando la solución estadística de Fariña, Arce y Novo (2002), empleando el estadístico kappa verdadero $(-\kappa)$. Para ello se registró como - 1 acuerdocuando había concordancia en la codificación entre el momento 1 y el momento 2 por un investigador, o bien cuando había concordancia en la codificación entre los dos investigadores. Se registró como - 1 desacuerdo- bien cuando había una discrepancia en la concordancia entre los dos momentos o bien cuando no había concordancia en la codificación entre los dos investigadores. Por último, se registró como -2 desacuerdos- cuando no había concordancia tanto en la codificación en el momento 1 y en el momento 2 y cuando tampoco la había entre los dos investigadores. El valor del estadístico kappa verdadera fue $\left({ }^{-} \kappa\right.$ $=1$ ) dada la facilidad a la hora de codificar las variables, lo que indica una buena concordancia inter e intra codificador y por consiguiente una buena fiabilidad en la codificación, tal y como sugiere Tversky (1977) al considerar consistentes aquellas evaluaciones con una puntuación de .80 o mayor.

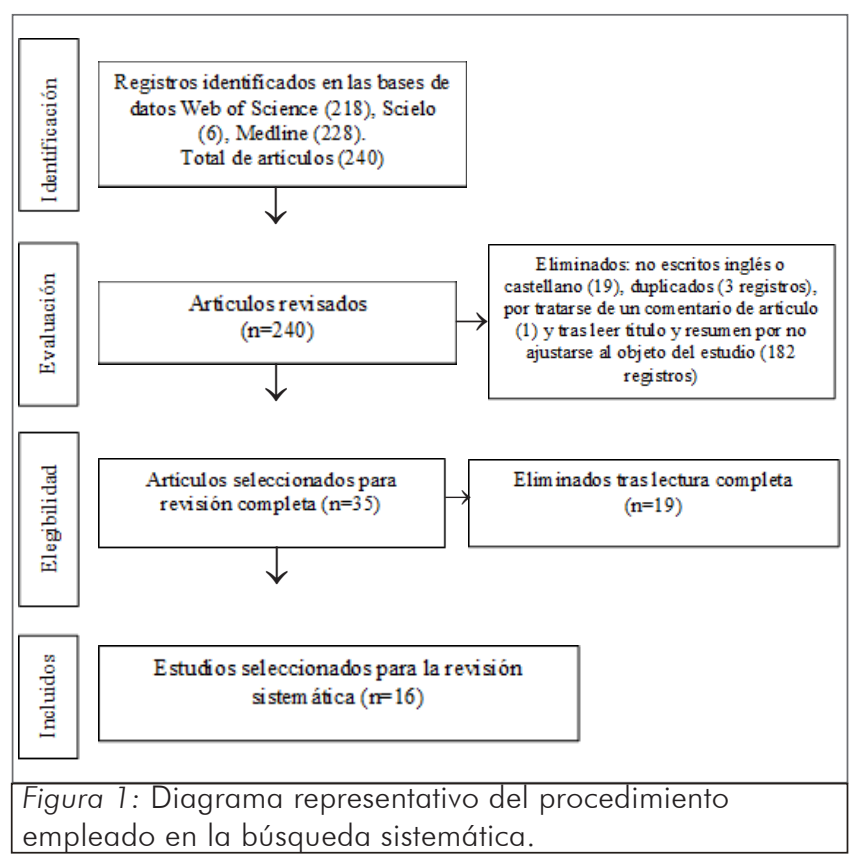


Tabla 1

Relación de estudios empleados en la presente revisión sistemática

\begin{tabular}{|c|c|c|c|c|}
\hline Estudio & Muestra & $\begin{array}{c}\text { Variables relacionadas con } \\
\text { el embarazo, parto y recién } \\
\text { nacido }\end{array}$ & $\begin{array}{l}\text { Resultados más } \\
\text { relevantes }\end{array}$ & Limitaciones del estudio \\
\hline $\begin{array}{l}\text { Brown ef } \\
\text { al. (2016) }\end{array}$ & $\begin{array}{c}\text { Australia. } N=337 \\
\text { madres/hijos. Edad 15- } \\
43 \text { años. } M=25.5 \text { años. } \\
\text { Cannabis }(N=12), \\
\text { Tabaco }(N=106) \text {, } \\
\text { Dual }(N=56), \text { No uso } \\
(N=158) .\end{array}$ & $\begin{array}{l}\text { Variables sociodemográficas. } \\
\text { Estresores maternos. Edad } \\
\text { gestacional. Peso al nacer. Parto } \\
\text { prematuro }(<37 \text { semanas). Bajo } \\
\text { peso al nacer }(<2500 \text { g). Tamaño } \\
\text { según edad gestacional }(P<10) \text {. }\end{array}$ & $\begin{array}{l}\text { Mayores problemas } \\
\text { psicosociales en } \\
\text { embarazadas con uso } \\
\text { de cannabis o co-uso. } \\
\text { Problemas perinatales } \\
\text { mayores en los grupos que } \\
\text { consumen cannabis. }\end{array}$ & $\begin{array}{l}\text { Uso de autoinformes sobre el } \\
\text { consumo. } \\
\text { No control del uso de alcohol } \\
\text { u otras drogas ilícitas durante } \\
\text { el embarazo }\end{array}$ \\
\hline $\begin{array}{l}\text { Chabarria } \\
\text { et al. } \\
\text { (2016) }\end{array}$ & $\begin{array}{l}\text { USA. No uso }(N=11769 \\
M=29.1 \pm 9.1 \text { años), } \\
\text { Tabaco ( } N=194 \\
M=28.2 \pm 9.5 \text { años), } \\
\text { Cannabis ( } N=58 \\
M=24 \pm 5.1 \text { años), } \\
\text { Dual ( } N=48, M=27 \pm \\
5.5 \text { años). }\end{array}$ & $\begin{array}{l}\text { Variables sociodemográficas. } \\
\text { Autoinforme consumo de drogas. } \\
\text { Asma, diabetes e hipertensión y } \\
\text { comorbilidades en salud mental. } \\
\text { Peso al nacer } \mathrm{P}<25 \text {. Perímetro } \\
\text { craneal } \mathrm{P}<25 \text {. Crecimiento fetal } \\
\mathrm{P}<25 \text {. Prematuridad. Test de } \\
\text { Apgar. }\end{array}$ & $\begin{array}{l}\text { Mayores riesgos perinatales } \\
\text { en los casos de consumo } \\
\text { dual cannabis-tabaco } \\
\text { frente al uso del cannabis. }\end{array}$ & $\begin{array}{l}\text { No control de otras variables } \\
\text { que pueden tener un efecto } \\
\text { en los resultados. Pérdida de } \\
\text { datos. Informes del paciente. } \\
\text { Informe de consumo de } \\
\text { marihuana menor a los datos } \\
\text { generales de consumo. Error } \\
\text { de tipo II. }\end{array}$ \\
\hline $\begin{array}{l}\text { Coleman- } \\
\text { Cowger } \\
\text { et al. } \\
(2017)\end{array}$ & $\begin{array}{l}\text { USA. Mujeres entre } \\
\text { 12-49 años. } N=8721 \\
\text { embarazadas ( } 433 \\
\text { dual). } N=488.497 \\
\text { no embarazadas } \\
(N=18622 \text { dual). }\end{array}$ & $\begin{array}{l}\text { Características demográficas. } \\
\text { Consumo de tabaco y/o } \\
\text { marihuana, frecuencia. } \\
\text { Dependencia a la marihuana. } \\
\text { Dependencia a la nicotina. Uso de } \\
\text { otras sustancias. }\end{array}$ & $\begin{array}{c}\text { Las embarazadas con } \\
\text { consumo dual presentan } \\
\text { más factores de riesgo que } \\
\text { las no embarazadas. No } \\
\text { hay diferencias en el perfil } \\
\text { entre consumo dual y uso de } \\
\text { cannabis, si hay diferencias } \\
\text { con el grupo de tabaco. } \\
\end{array}$ & $\begin{array}{l}\text { Autoinformes. Estudio } \\
\text { transversal. No se tienen en } \\
\text { cuenta variables ambientales } \\
\text { cambiantes en los años de } \\
\text { estudio. No se aportan datos } \\
\text { por trimestre de embarazo. }\end{array}$ \\
\hline $\begin{array}{l}\text { Coleman- } \\
\text { Cowger } \\
\text { et al. } \\
(2018)\end{array}$ & $\begin{array}{c}\text { USA. } N=500 . \text { Dual } \\
\text { ( } N=45, M=26.7, \\
D T=4.8 \text { años). Tabaco } \\
\text { ( } N=39, M=27.9, \\
D T=4.4 \text { años), } \\
\text { Cannabis }(N=60, \\
M=27.3, D T=4.9 \text { años }) \\
\text { No consumo }(N=354, \\
M=28.2, D T=5.4) .\end{array}$ & $\begin{array}{l}\text { Variables demográficas e historia } \\
\text { obstétrica. Uso de drogas. Edad } \\
\text { gestacional, peso y longitud } \\
\text { al nacer, circunferencia de la } \\
\text { cabeza, test de Apgar, cuidados } \\
\text { intensivos y presencia o defectos } \\
\text { de nacimiento. }\end{array}$ & $\begin{array}{l}\text { Co-uso }(9 \%) \text { durante el } \\
\text { embarazo. Co-uso más } \\
\text { probabilidades de presentar } \\
\text { un perímetro craneal más } \\
\text { pequeño y defectos en } \\
\text { el neonato. En el resto de } \\
\text { variables relacionadas con } \\
\text { el nacimiento no se dieron } \\
\text { diferencias estadísticamente } \\
\text { significativas. }\end{array}$ & $\begin{array}{c}\text { Tamaño de la muestra } \\
\text { pequeño. No se recogen } \\
\text { datos acerca de los patrones } \\
\text { de consumo ni del trimestre } \\
\text { del embarazo. }\end{array}$ \\
\hline $\begin{array}{l}\text { De } \\
\text { Genna et } \\
\text { al. (2018) }\end{array}$ & $\begin{array}{l}\text { USA. } N=603 \text { madres/ } \\
\text { hijos. Madres: }(18-42 \\
\text { años, } M=23, D T=4.0) \text {. } \\
\text { Hijos: ( } M=23, D T=0.07 \\
\text { años). }\end{array}$ & $\begin{array}{l}\text { Consumo de drogas. Depresión, } \\
\text { ansiedad y agresividad en las } \\
\text { madres } 16 \text { años tras el parto. } \\
\text { Descendencia: Salud mental. } \\
\text { Trastorno por consumo de drogas. } \\
\text { Cl. Atención e impulsividad. } \\
\text { Problemas externalizantes e } \\
\text { internalizantes. }\end{array}$ & $\begin{array}{l}\text { Se identifican tres patrones } \\
\text { de consumo conjunto en } \\
\text { mujeres cuando estaban } \\
\text { embarazadas y } 16 \text { años } \\
\text { después. Más riesgo de } \\
\text { trastornos por consumo de } \\
\text { sustancias en los hijos de } \\
\text { madres que presentaban } \\
\text { consumo dual durante el } \\
\text { embarazo. Controlando el } \\
\text { sexo, masculino, mayores } \\
\text { problemas internalizantes y } \\
\text { menor logro académico. }\end{array}$ & $\begin{array}{l}\text { Muestra recogida en la } \\
\text { década de los } 80 \text {. Tamaño } \\
\text { de la muestra pequeño } \\
\text { en el grupo de consumo } \\
\text { dual. No control de otras } \\
\text { variables que pueden influir } \\
\text { en la relación de consumo } \\
\text { conjunto materno y de la } \\
\text { descendencia. }\end{array}$ \\
\hline $\begin{array}{l}\text { Eiden, } \\
\text { Schuetze } \\
\text { et al. } \\
(2018)\end{array}$ & $\begin{array}{l}\text { USA. } N=247 \text { madres/ } \\
\text { hijos. Madres: } 18-39 \\
\text { años. Dual }(N=97 \text {, } \\
M=23.8, D T=4.7) \text {, } \\
\text { Tabaco }(N=81, \\
M=24.9, D T=5.19) \\
\text { No consumo }(N=69, \\
M=24.4, D T=5.02) .\end{array}$ & $\begin{array}{l}\text { Biomarcadores consumo } \\
\text { Sociodemográficas. Arritmia } \\
\text { sinusal respiratoria. Problemas } \\
\text { de regulación maternal. Riesgo } \\
\text { psicológico materno. Sensibilidad } \\
\text { materna. Regulación emocional.. }\end{array}$ & $\begin{array}{l}\text { El consumo conjunto afecta } \\
\text { a la regulación emocional } \\
\text { del niño, siendo menor a los } \\
9 \text { meses y a los } 24 \text { meses. } \\
\text { Sensibilidad más baja en las } \\
\text { madres en las interacciones } \\
\text { de juego con los bebes. }\end{array}$ & $\begin{array}{l}\text { Situación de laboratorio } \\
\text { para medir la regulación } \\
\text { emocional. No se midió el } \\
\text { uso de cannabis después del } \\
\text { embarazo. }\end{array}$ \\
\hline $\begin{array}{l}\text { Eiden, } \\
\text { Zhao et } \\
\text { al. (2018) }\end{array}$ & $\begin{array}{c}\text { USA. } 247 \text { madres/ } \\
\text { hijos. Edad 18-39 años } \\
(M=24.09, D T=5.0) . \\
\text { Dual }(N=103) \text {, Tabaco } \\
(N=75), \text { No consumo } \\
(N=69) .\end{array}$ & $\begin{array}{c}\text { Autoinformes y biomarcadores } \\
\text { consumo tabaco y/o cannabis en } \\
\text { el embarazo. Exposición posnatal } \\
\text { al tabaco. Uso cannabis posnatal. } \\
\text { Problemas de conducta en los } \\
\text { niños-as. }\end{array}$ & $\begin{array}{c}\text { El uso de cannabis predijo } \\
\text { problemas de conducta a } \\
\text { los } 2 \text { años y estos a su vez } \\
\text { un mayor uso de cannabis } \\
\text { un año después. } \\
\text { No hay diferencias en } \\
\text { problemas conductuales, } \\
\text { si bien en el caso de las } \\
\text { niñas, aquéllas expuestas } \\
\text { al tabaco presentan más } \\
\text { problemas internalizantes. }\end{array}$ & $\begin{array}{l}\text { Tamaño de la muestra. Uso } \\
\text { de informes de la madre } \\
\text { acerca del consumo de } \\
\text { cannabis postnatal y de los } \\
\text { problemas de conducta. } \\
\text { Selección de la muestra. }\end{array}$ \\
\hline
\end{tabular}




\section{Tabla 1 (Continuación)}

Relación de estudios empleados en la presente revisión sistemática

\begin{tabular}{|c|c|c|c|c|}
\hline Estudio & Muestra & $\begin{array}{c}\text { Variables relacionadas con } \\
\text { el embarazo, parto y recién } \\
\text { nacido }\end{array}$ & $\begin{array}{l}\text { Resultados más } \\
\text { relevantes }\end{array}$ & Limitaciones del estudio \\
\hline $\begin{array}{l}\text { Godleski } \\
\text { et al. } \\
(2018)\end{array}$ & $\begin{array}{c}\text { USA } N=247 \text { madres/ } \\
\text { hijos. Edad 18-39 } \\
\text { años }(M=24.09) . \\
\text { Dual }(N=97), \text { Tabaco } \\
(N=81), \text { No uso tabaco } \\
(N=69)\end{array}$ & $\begin{array}{l}\text { Uso prenatal y posnatal de } \\
\text { sustancias. Factores demográficos } \\
\text { de riesgo. Sensibilidad materna. } \\
\text { Lactancia. Predisposición de la } \\
\text { madre a la agresividad. Problemas } \\
\text { de conducta externalizantes. }\end{array}$ & $\begin{array}{l}\text { No existe relación directa } \\
\text { entre consumo dual y } \\
\text { fumar tabaco y problemas } \\
\text { externalizantes. No hay } \\
\text { diferencias en función } \\
\text { del sexo del neonato y } \\
\text { el grupo de consumo/no } \\
\text { consumo en problemas } \\
\text { externalizantes a los } 24 \\
\text { meses. A los } 36 \text { meses los } \\
\text { varones presentan mayores } \\
\text { problemas externalizantes } \\
\text { en el grupo de consumo } \\
\text { conjunto y no consumo. }\end{array}$ & $\begin{array}{l}\text { Falta de un grupo de } \\
\text { consumo solo de marihuana. } \\
\text { Características de la muestra } \\
\text { al ser madres con bajos } \\
\text { niveles socioeducativos. Uso } \\
\text { de autoinformes maternos } \\
\text { sobre el uso de cannabis } \\
\text { posnatal. Exclusión de madres } \\
\text { con uso abusivo de alcohol. }\end{array}$ \\
\hline $\begin{array}{l}\text { Gould et } \\
\text { al. (2017) }\end{array}$ & $\begin{array}{l}\text { Australia. Médicos } \\
\text { generales y } \\
\text { obstetras }(N=378 \text {, } \\
\text { Hombres }=142 \text {, } \\
\text { Mujeres }=235)\end{array}$ & $\begin{array}{c}\text { Frecuencia con la que preguntan } \\
\text { a embarazadas sobre el uso de } \\
\text { cannabis, cannabis y tabaco, } \\
\text { e-cigarrillo con y sin nicotina, } \\
\text { tabaco de mascar y exposición al } \\
\text { humo. }\end{array}$ & $\begin{array}{c}\text { Un } 37.7 \% \text { de los } \\
\text { profesionales sanitarios } \\
\text { preguntan de forma } \\
\text { frecuente acerca del } \\
\text { consumo dual y un } 58 \% \\
\text { sobre el uso del cannabis. }\end{array}$ & $\begin{array}{l}\text { Bajas tasas de respuesta. } \\
\text { Sobreestimación de los } \\
\text { resultados al contestar } \\
\text { aquellos médicos que más } \\
\text { les puede interesar el estudio. } \\
\text { Uso de autoinformes sobre } \\
\text { consumo. No se preguntó } \\
\text { acerca de otras formas de } \\
\text { consumir tabaco. }\end{array}$ \\
\hline $\begin{array}{l}\text { Gray et } \\
\text { al. (2010) }\end{array}$ & $\begin{array}{l}\text { USA. } N=86 \text { madres } / \text { hijos. } \\
\text { No uso cannabis } \\
(N=48, M=24.3 \\
D T=5.2), \text { Cannabis } \\
(N=38,(M=24.04 \\
D T=5.1), \text { de los cuales } \\
(N=33,86.8 \%) \text { usan } \\
\text { también tabaco. }\end{array}$ & $\begin{array}{c}\text { Consumo de sustancias. Variables } \\
\text { sociodemográficas. Sexo. Edad } \\
\text { gestacional. Peso y longitud al } \\
\text { nacer. Perímetro craneal. Test de } \\
\text { Apgar. }\end{array}$ & $\begin{array}{l}\text { Reducción del crecimiento } \\
\text { fetal tras controlar el } \\
\text { consumo de tabaco. } \\
\text { El grupo de tabaco y } \\
\text { cannabis menor peso, } \\
\text { longitud y circunferencia } \\
\text { de la cabeza, no habiendo } \\
\text { diferencias en edad } \\
\text { gestacional y test de Apgar. } \\
\text { En el grupo de exposición al } \\
\text { cannabis no hay diferencias. }\end{array}$ & $\begin{array}{l}\text { Mayor número de toma de } \\
\text { muestras de saliva antes del } \\
\text { embarazo y entre aquellas } \\
\text { que informaron que no } \\
\text { consumían. Limitaciones a } \\
\text { la hora de identificar el uso } \\
\text { de cannabis a través del } \\
\text { meconio si el consumo se ha } \\
\text { producido en los dos primeros } \\
\text { trimestres. }\end{array}$ \\
\hline $\begin{array}{l}\text { Leemaqz } \\
\text { et al. } \\
(2016)\end{array}$ & $\begin{array}{c}\text { Australia, Nueva } \\
\text { Zelanda, Irlanda, Reino } \\
\text { Unido. } \\
N=5588 \text { embarazadas } \\
(M=28.6 \pm 0.1 \\
\text { años). Casos sin } \\
\text { complicaciones } 4074 \\
\text { y con complicaciones } \\
\text { en el embarazo } 1514 .\end{array}$ & $\begin{array}{l}\text { Variables sociodemográficas. } \\
\text { Historial médico y familiar. Dieta. } \\
\text { Estilo de vida. Consumo de tabaco } \\
\text { y cannabis y de otras drogas. } \\
\text { Parto prematuro espontáneo } \\
<37 \text { semanas. Peso al nacer } \\
\text { P<10. Preeclampsia. Diabetes } \\
\text { gestacional. }\end{array}$ & $\begin{array}{c}\text { El consumo de marihuana } \\
\text { con independencia del } \\
\text { consumo o no de tabaco } \\
\text { incrementa el riesgo de } \\
\text { prematuridad. El consumo } \\
\text { dual aumenta más del } \\
\text { doble la probabilidad de } \\
\text { prematuridad. }\end{array}$ & $\begin{array}{l}\text { Uso de autoinformes acerca } \\
\text { del consumo. Muestra } \\
\text { formada sólo por madres } \\
\text { primerizas. }\end{array}$ \\
\hline $\begin{array}{l}\text { Massey et } \\
\text { al. (2018) }\end{array}$ & $\begin{array}{c}\text { USA } \\
1191 \text { madres/hijos. } \\
\text { Tabaco }(N=608), \\
\text { Marihuana }(N=273), \\
\text { Dual }(N=230) . \text { Niños } \\
(N=654) \text { y niñas } \\
(N=537)\end{array}$ & $\begin{array}{l}\text { Uso de drogas. Sexo del recién } \\
\text { nacido. Edad gestacional. Peso al } \\
\text { nacer. }\end{array}$ & $\begin{array}{l}\text { Uso de tabaco y marihuana } \\
\text { se asocia con bajo peso } \\
\text { al nacer. El consumo dual } \\
\text { no se asocia con el peso } \\
\text { al nacer ni con la duración } \\
\text { de la gestación. No hay } \\
\text { diferencias en función del } \\
\text { sexo del recién nacido de } \\
\text { madres con consumo dual } \\
\text { en peso al nacer, pero sí en } \\
\text { el grupo de marihuana. }\end{array}$ & $\begin{array}{l}\text { Muestras poco representativas } \\
\text { en consumos elevados. No } \\
\text { considerar otras variables } \\
\text { que afectan al peso al nacer } \\
\text { o a la edad gestacional. } \\
\text { No considerar el momento } \\
\text { del consumo. Sesgos en la } \\
\text { selección de la muestra que } \\
\text { consumía alcohol. }\end{array}$ \\
\hline $\begin{array}{l}\text { Molnar et } \\
\text { al. (2018) }\end{array}$ & $\begin{array}{c}\text { USA. } N=50 \text { madres/ } \\
\text { hijos. } \\
\text { Tabaco }(N=19) \text {, Dual } \\
(N=19), \text { Grupo control } \\
\text { no consumo }(N=12) .\end{array}$ & $\begin{array}{l}\text { Variables sociodemográficas. } \\
\text { Uso de drogas mediante } \\
\text { autoinforme y biomarcadores pre } \\
\text { y posnatal. Edad gestacional. } \\
\text { Inmunoglobina-A. }\end{array}$ & $\begin{array}{l}\text { El uso de tabaco y el } \\
\text { consumo dual se relaciona } \\
\text { con una mayor secreción } \\
\text { de la Inmunoglobulina A en } \\
\text { niños entre } 5 \text { y } 6 \text { años. }\end{array}$ & $\begin{array}{l}\text { Dificultad de medir el } \\
\text { consumo de drogas. Muestra: } \\
\text { pequeña y muy específica } \\
\text { (mujeres jóvenes y de bajos } \\
\text { ingresos). Evaluaciones } \\
\text { del uso de cigarrillo más } \\
\text { exhaustivas que las de } \\
\text { consumo de cannabis. }\end{array}$ \\
\hline
\end{tabular}


Tabla 1 (Continuación)

Relación de estudios empleados en la presente revisión sistemática

\begin{tabular}{|c|c|c|c|c|}
\hline Estudio & Muestra & $\begin{array}{c}\text { Variables relacionadas con } \\
\text { el embarazo, parto y recién } \\
\text { nacido }\end{array}$ & $\begin{array}{l}\text { Resultados más } \\
\text { relevantes }\end{array}$ & Limitaciones del estudio \\
\hline $\begin{array}{l}\text { Saurel- } \\
\text { Cubizolles } \\
\text { et al. } \\
\text { (2014) }\end{array}$ & $\begin{array}{c}\text { Francia. } N=13531 \text { no } \\
\text { consumidoras. } N=158 \\
\text { cannabis de las cuales } \\
\text { el } 71.6 \% \text { consumo } \\
\text { dual. }\end{array}$ & $\begin{array}{l}\text { Variables sociodemográficas. Uso } \\
\text { de drogas. Edad gestacional. } \\
\text { Parto prematuro, espontáneo o } \\
\text { por prescripción médica. Tamaño } \\
\text { según edad gestacional P<10. } \\
\text { Peso al nacer. }\end{array}$ & $\begin{array}{l}\text { Baja prevalencia de } \\
\text { consumo de cannabis en } \\
\text { embarazadas. } \\
\text { El uso del cannabis se } \\
\text { relaciona con partos } \\
\text { prematuros. }\end{array}$ & $\begin{array}{l}\text { Autoinforme acerca del } \\
\text { uso de sustancias, como } \\
\text { el cannabis, no uso de } \\
\text { biomarcadores. }\end{array}$ \\
\hline $\begin{array}{l}\text { Schuetze } \\
\text { et al. } \\
\text { (2018) }\end{array}$ & $\begin{array}{c}\text { USA. } N=247 \text { ( } M=25.39 \\
D T=4.99 \text { años). } \\
173 \text { niños expuestos } \\
\text { al tabaco y } 74 \text { no } \\
\text { expuestos a sustancias. }\end{array}$ & $\begin{array}{c}\text { Biomarcadores para determinar el } \\
\text { uso de sustancias. } \\
\text { Sentimiento de ira y estrés en } \\
\text { las madres pre y posnatal. } \\
\text { Circunferencia de la cabeza. } \\
\text { Peso y longitud al nacer. Edad } \\
\text { gestacional. Reactividad del } \\
\text { recién nacido. }\end{array}$ & $\begin{array}{l}\text { Problemas en el crecimiento } \\
\text { fetal, siendo mayor en } \\
\text { el grupo de consumo } \\
\text { conjunto, afectando } \\
\text { principalmente a los hijos } \\
\text { varones. No existe relación } \\
\text { directa entre consumo y } \\
\text { reactividad/regulación en } \\
\text { el recién nacido, pero sí a } \\
\text { través de vías indirectas. }\end{array}$ & $\begin{array}{l}\text { Determinar el consumo } \\
\text { real de sustancias en las } \\
\text { mujeres embarazadas. No } \\
\text { tener datos acerca de la } \\
\text { salud psicológica materna. } \\
\text { La medida de la regulación } \\
\text { infantil solo se midió mediante } \\
\text { una única tarea. }\end{array}$ \\
\hline $\begin{array}{l}\text { Stroud et } \\
\text { al. (2018) }\end{array}$ & $\begin{array}{c}\text { USA. } 111 \text { madres/hijos } \\
\text { ( } M=25, D T=5 \text { años). } \\
\text { No uso ( } N=42, M=25, \\
D T=6 \text { años), Co-uso } \\
(N=24, M=25, D T=4 \\
\text { años), Tabaco ( } N=45, \\
M=24, D T=4 \text { años). } \\
\text { Hijos ( } N=101,51 \text { niñas y } \\
60 \text { niños). }\end{array}$ & $\begin{array}{l}\text { Biomarcadores para determinar } \\
\text { el consumo de sustancias pre y } \\
\text { posnatal. Variables referidas a } \\
\text { los menores: Autorregulación, } \\
\text { Necesidad de cuidado externo } \\
\text { para calmarse, Letargo y Atención. }\end{array}$ & $\begin{array}{l}\text { Recién nacidos de madres } \\
\text { con consumo conjunto } \\
\text { mayores dificultades para } \\
\text { calmarse, mayor necesidad } \\
\text { de atención externa, menor } \\
\text { atención a estímulos y mayor } \\
\text { letargo frente a los nacidos } \\
\text { de madres fumadoras de } \\
\text { tabaco. }\end{array}$ & $\begin{array}{l}\text { Tamaño de la muestra, } \\
\text { principalmente en el grupo } \\
\text { de consumo conjunto. No } \\
\text { disponer de un grupo de } \\
\text { solo consumo de marihuana. } \\
\text { No controlar la dosis de } \\
\text { consumo. }\end{array}$ \\
\hline
\end{tabular}

\section{RESULTADOS}

\section{•CARACTERÍSTICAS DE LOS ESTUDIOS.}

La Tabla 1 recoge las características más relevantes de los artículos incluidos en la revisión sistemática como fueron la descripción de la muestra, variables relacionadas con el embarazo, parto y recién nacido, resultados más relevantes y limitaciones de los estudios.

De los 16 trabajos analizados 12 son realizados en Estados Unidos, dos en Australia, uno en Francia y uno de ellos, en concreto el de Leemaqz et al. (2016), se llevó a cabo con muestras de diferentes países. Con respecto al tamaño de la muestra, este suele ser muy diferente, siendo los trabajos con muestras más grandes procedentes de encuestas a nivel nacional como el de Coleman-Cowger et al. (2017) en Estados Unidos, o el de SaurelCubizolles et al. (2014) a través de una muestra representativa de todos los nacimientos producidos en la primera semana de marzo de 2010 en Francia. En concreto, los tamaños muestrales de los grupos de consumo dual son pequeños, siendo el de Coleman-Cowger et al. (2018) el que presenta una muestra más numerosa con 433 gestantes con consumo dual.

Con respecto a las edades de las embarazadas solo el trabajo de ColemanCowger et al. (2017) incluye a madres menores de 18 años, si bien el realizado por SaurelCubizolles et al. (2014) incluye un grupo de 25 años o menos pero no especifica cuál es la edad menor. Con respecto a la media de edad de las gestantes suele situarse alrededor de los 24-25 años, salvo los trabajos de Chabarría et al. (2016) y Coleman-Cowger et al. (2018) con medias entre 27 y 29 años. Por último reseñar que 10 de los trabajos han incluido tanto a la madre como a la descendencia, cinco trabajos únicamente a mujeres embarazadas y un trabajo, el de Gould et al. (2017) a personal médico. 


\section{- EMBARAZADAS CON CONSUMO DUAL CANNABIS-TABACO: PERFIL PSICOSOCIAL $Y$ RELACIÓN CON OTRAS VARIABLES.}

El perfil de la embarazada consumidora de cannabis-tabaco se caracteriza, según Coleman-Cowger et al. (2017) por tener una edad entre 18 y 25 años, de raza negra no hispana, bajo nivel educativo y económico, con niveles de dependencia hacia la marihuana y hacia la nicotina, consumiendo entre 6 y 15 cigarrillos por día frente a las mujeres no embarazadas. A la hora de comparar el perfil de mujeres gestantes que presentaban algún tipo de consumo, ya sea solo marihuana, solo tabaco o consumo conjunto, no hay diferencias entre consumidoras de marihuana y consumo conjunto. En cambio sí existen diferencias entre consumidoras de tabaco y consumo dual, resultando que las consumidoras de ambas sustancias presentan un mayor uso del tabaco, de alcohol y de drogas ilegales, con mayor presencia de mujeres de raza negra no hispanas e hispanas, entre 12 y 17 años, habiendo consumido principalmente en el primer trimestre del embarazo. Además, según Coleman-Cowger et al. (2018) la mayoría de ellas no tenían la intención de quedarse embarazadas.

Para Brown et al. (2016) las madres consumidoras de cannabis o de éste junto al tabaco suelen tener hijos a una menor edad, nivel educativo más bajo y desempleo. Para Stroud et al. (2018) presentan un nivel socioeconómico más bajo, están expuestas a ambientes con humo de tabaco y manifiestan mayor sintomatología depresiva frente a gestantes no consumidoras.

Saurel-Cubizolles et al. (2014) relacionan el consumo de cannabis con un mayor uso de tabaco y de alcohol, ser francesa, soltera, primer embarazo, bajo nivel educativo y bajos ingresos. Para Gray et al. (2010) las embarazadas que consumen cannabis suelen consumir también tabaco en la misma cantidad frente a las que solo fuman tabaco. Según Schuetze, Eiden, Colder, Huestis y Leonard (2018) las que presentan consumo dual tienen mayores problemas socioeconómicos, menor probabilidad de estar casadas o de vivir en convivencia.

Brown et al. (2016) apuntan a que las mujeres con consumo dual presentan un mayor número de sucesos estresantes y problemas psicosociales y un menor compromiso y frecuencia en el seguimiento médico del embarazo. En cuanto al personal sanitario, destaca la baja frecuencia a la hora de preguntar a las embarazadas sobre el uso del cigarrillo electrónico, tabaco de mascar, exposición al humo y el cannabis. Según Gould et al. (2017) sólo un $37,7 \%$ preguntaba acerca del consumo conjunto cannabis-tabaco a las gestantes.

\section{-EFECTOS DEL CONSUMO DUAL CANNABIS- TABACO SOBRE EL EMBARAZO Y EL DESARROLLO PRE, PERI Y POSTNATAL.}

Los resultados de la revisión sistemática muestran diferencias en cuanto al efecto del consumo dual cannabis-tabaco en los parámetros relacionados con el desarrollo pre, peri y postnatal.

En este sentido parece existir una relación entre el consumo dual y el perímetro craneal del recién nacido, siendo este más pequeño (Schuetze et al., 2018). Para Coleman et al. (2018) existirían 5.7 veces más probabilidades de un perímetro craneal inferior en niños de madres con consumo dual frente a las que no consumen, habiendo un mayor número de casos con percentiles craneales inferiores al percentil 25 (Chabarria et al., 2016). Por el contrario, Gray et al. (2010) apuntan que a pesar de ser inferior, éste se encuentra dentro de las medidas de normalidad.

En cuanto al peso al nacer, Brown et al. (2016) señalan que los recién nacidos de consumidoras de cannabis y de consumo conjunto pesan una media de 565 gramos menos y tienen más probabilidades de bajo peso ( $<2500$ gramos), resultados similares a los encontrados por Schuetze et al. (2018) y Saurel-Cubizolles et al. (2014), en especial en el grupo de consumo dual. En cambio, Coleman-Cowger et al. (2018) y Gray et al. (2010) no encuentran diferencias significativas en el peso al nacer. Si bien, en los tres grupos de consumidoras existe una relación con un menor peso al nacer, principalmente en el grupo de 
consumidoras de tabaco y en el grupo de uso de cannabis, siendo aproximadamente un $18 \%$ más bajo el peso de lo esperado (Masey et al., 2018), no habiendo un efecto sumativo en el grupo de consumidoras de ambas sustancias con independencia del sexo del recién nacido.

La longitud al nacer ha sido otro de los parámetros estudiados, siendo menor en el grupo de consumo dual frente a los grupos de consumo de una sola sustancia (Chabarría et al., 2016; Saurel-Cubizolles et al., 2014; Schuetze et al., 2018). Para Gray et al. (2010) el uso del cannabis se relaciona con una menor longitud del recién nacido pero dentro de los parámetros normales, incluso después de que los datos se controlaran para la coexposición al tabaco. Coleman-Cowger et al. (2018) en cambio no encuentra diferencias significativas entre grupos de consumidoras.

Leemaqz et al. (2016) relacionan el consumo de marihuana y tabaco a las 20 semanas de gestación con una menor edad gestacional y con prematuridad. La prematuridad se asocia con el consumo de marihuana, resultando que un $36.4 \%$ dio a luz antes de las 28 semanas y un $63.6 \%$ antes de las 32 semanas, siendo el riesgo mayor si el consumo se ha dado durante el embarazo o en los 3 meses anteriores, diferencias que se mantienen con independencia de si presentan consumo dual. Por su parte, Saurel-Cubizolles et al. (2014) apunta a que las consumidoras solo de cannabis presentaron con más frecuencia partos antes de las 32 semanas de gestación, mientras que aquéllas que además consumían tabaco presentaban mayores probabilidades de dar a luz antes de la semana 37. Para Brown et al. (2016) el $51 \%$ de los recién nacidos de madres consumidoras de cannabis y de consumo conjunto presentaban mayor prematuridad frente al grupo de consumo sólo de tabaco, donde el porcentaje era del $30 \%$ y del $24 \%$ en el grupo de no consumidoras.

En cuanto a la edad gestacional, lo cual no implica prematuridad, ésta es menor en embarazadas con consumo dual (Chabarria et al., 2016; Saurel-Cubizolles et al., 2014; Schuetze et al., 2018), habiendo relación con el uso de la marihuana (Leemaqz et al., 2016), no habiendo diferencias entre los tres grupos de consumidoras según Coleman-Cowger et al. (2018), Gray et al. (2010) y Masey et al. (2018). Para Schuetze et al. (2018) existe una asociación entre consumo de tabaco y/o marihuana y el crecimiento fetal, siendo esta asociación más fuerte en los casos de consumo dual, afectando más a los niños que a las niñas.

Los defectos en el recién nacido son mayores en los grupos de consumo de cannabis (Brown et al., 2016), siendo tres veces mayor en el grupo de consumo dual (ColemanCowger et al., 2018). También se observa una mayor presencia de problemas perinatales en los grupos con consumo de cannabis frente al grupo de uso de tabaco (Brown et al., 2016).

En otros parámetros analizados no hay diferencias entre grupos, como es el caso de las puntuaciones en el test de Apgar (ColemanCowger et al., 2018; Gray et al., 2010), hipertensión arterial y diabetes gestacional (Leemaqz et al., 2016), en la medicalización del parto (Saurel-Cubizolles et al., 2014) y en la necesidad de cuidados intensivos neonatales (Coleman-Cowger et al., 2018). En el caso de la preeclampsia no existe relación con el consumo dual (Leemaqz et al., 2016), mientras que Chabarria et al. (2016) sí encuentra relación, al igual que con la presencia de asma en la madre.

Otros indicadores evaluados han sido la presencia de problemas externalizantes e internalizantes. En concreto, Godleski, Shisler, Eiden y Huestis (2018) no encuentran relación directa entre el consumo dual o uso de tabaco y la presencia de problemas externalizantes a los 24 y 36 meses, si bien se observa una relación indirecta sobre estos problemas y la calidez y sensibilidad materna con el consumo dual. Estos autores señalan también una relación significativa con la duración de la lactancia materna, siendo menor en madres con consumo conjunto cannabis-tabaco. Para Eiden, Zhao et al. (2018) no hay diferencias entre mujeres que consumieron alguna de estas sustancias durante la gestación y las no consumidoras en relación a problemas conductuales en los hijos, si bien sí existen diferencias en función del sexo, siendo las niñas de madres consumidoras de tabaco las que presentan mayores problemas internalizantes. 
Para Stroud et al. (2018) existe relación entre el consumo de tabaco y marihuana en la autorregulación y a la hora de estar calmado en estados de alerta en recién nacidos de un mes, tanto en consumidoras de tabaco como de cannabis y tabaco, presentando niveles más bajos de autorregulación y mayor necesidad de calmarse el grupo de consumo dual, incluso frente al grupo de consumo solo de tabaco. Estas diferencias son mayores en ambos sexos en el grupo de consumo dual frente al grupo de no consumidoras, mientras que al compararlo con el grupo de tabaco se da un mayor efecto en las niñas. En relación a la atención a estímulos visuales y auditivos, los recién nacidos de madres con consumo dual presentaron niveles atencionales más bajos frente al grupo de consumo de tabaco, si bien estas diferencias no fueron estadísticamente significativas en función del sexo. El letargo fue mayor significativamente en los grupos de consumidoras de cannabis y/o tabaco frente al grupo de no consumo en el caso de las niñas. En cambio, no hubieron diferencias entre los recién nacidos de madres con consumo conjunto y con consumo solo de tabaco, si bien el primer grupo presentaba niveles de letargia mayores.

Eiden, Schuetze, Shisler y Huestis (2018) encuentran la regulación automática a los 9 meses y de la emoción a los 24 meses más afectada en el grupo de consumo dual y uso de tabaco frente al grupo de no consumidoras. Además, se ven más afectadas las interacciones en el juego entre la madre y el bebé, habiendo relación con una mayor presencia de problemas psicológicos en la madre. Por su parte, Schuetze et al. (2018) apuntan que las madres consumidoras de cannabis informan de mayor agresividad pre y postnatal, dándose en el grupo de consumo dual una asociación entre estrés materno y afecto negativo hacia el hijo a los dos meses. Estos mismos autores señalan la existencia de una relación indirecta en el grupo de consumo dual entre el crecimiento fetal y una menor regulación en el niño. Por su parte, no existe relación entre el sexo del bebe y la reactividadregulación entre los diferentes grupos de consumidoras.
De Genna, Goldschmidt, Richardson, Cornelius y Day (2018) consideran que el consumo conjunto en las madres predice el consumo cannabis tabaco en los hijos, especialmente en los varones, además del uso de otras drogas a los 22 años.

El estudio de Molnar et al. (2018) relaciona el consumo de cannabis y/o tabaco con la inmunoglobina $A$ en niños entre 5 y 6 años, observándose mayores niveles de inmunoglobina $A$ secretora en los casos en los que las madres habían consumido tabaco y cannabis-tabaco prenatal y postnatal. La presencia mayor de esta hormona se relaciona con el asma (Tinuoye, Pell, y Mackay, 2013) y con un peor funcionamiento pulmonar (Wang et al., 2015).

\section{DISCUSIÓN Y CONCLUSIONES}

El consumo de drogas legales e ilegales durante el embarazo ha sido abordado en un gran número de estudios. Entre estos destacan los trabajos relacionados con el tabaco y el cannabis de forma independiente, y en menor medida el consumo conjunto de ambas sustancias, en especial en el caso de las embarazadas si se tiene en consideración el escaso número de trabajos realizados, 16 en total, incluidos en la presente revisión sistemática.

Por otro lado, resulta difícil generalizar los resultados si se atiende a las características de la muestra de estos trabajos, como por ejemplo grupos de consumo dual con muestras pequeñas y mayoritariamente procedentes de Estados Unidos.

Con respecto al perfil psicosocial, las gestantes con consumo conjunto cannabistabaco se caracterizan por una mayor presencia de factores de riesgo psicosociales frente al grupo de embarazadas consumidoras de tabaco y no consumidoras, presentando consumo antes de quedarse embarazadas, siendo el primer trimestre el periodo de mayor consumo. En este sentido, Oga et al. (2017) apuntan que las gestantes que continúan consumiendo durante el embarazo cumplen en muchas ocasiones con los criterios de dependencia al cannabis o riesgo de ello 
según los criterios del DSM-IV, y una mayor dificultad para dejar de consumir (Peters, Budney y Carrol, 2012).

Dada la prevalencia del consumo conjunto cannabis-tabaco en embarazadas y el posible efecto aditivo de ambas sustancias (El Marroun et al., 2009), resulta necesario la inclusión en los estudios de un grupo específico de consumo dual, tal y como así apuntan Godleski et al. (2018), con el objetivo de conocer cómo ambas sustancias en combinación afectan a la gestación. Según los resultados hallados en el presente trabajo parece haber un efecto aditivo del consumo de las dos sustancias en algunos indicadores - marcadores pre, peri y postnatales, sin embargo se requieren un mayor número de estudios que permitan determinar este efecto, definiendo bien los grupos de consumo dual en relación a la cantidad y tipo de consumo.

Algunas de las limitaciones de los trabajos analizados están relacionadas con el diseño inicial del estudio, ya que no contemplaban un grupo deconsumocannabis-tabaco, incluyendo este grupo a posteriori dada la prevalencia de consumo dual. Otras, se relacionan con el tamaño de la muestra, la evaluación del consumo mediante autoinformes, no controlar variables relacionadas con el consumo, como frecuencia, cantidad, pureza, forma de consumo, y no controlar otras variables de tipo genético, de salud o sociodemográficas. Por último, reseñar como otras limitaciones el escaso número de trabajos incluidos en la presente revisión, lo que a su vez dificulta el poder realizar un meta-análisis.

Estos resultados permiten recoger toda una serie de orientaciones de cara a la intervención con embarazadas con consumo dual de cannabis y tabaco, intervenciones holísticas en las cuales se ha de incluir a la pareja y variables contextuales que rodean a la mujer gestante (Brown et al., 2016), así como factores de carácter social relacionados con el inicio y el mantenimiento del consumo (Huizink, 2009).

Estas intervenciones suponen involucrar a diferentes administraciones o servicios, principalmente de salud y servicios sociales, diseñando intervenciones específicas y posibilitando canales de comunicación entre administraciones.

Con respecto al ámbito sanitario, considerando la vulnerabilidad de estos periodos de desarrollo, resulta necesario concienciar y formar a los profesionales de la salud de los efectos negativos del consumo conjunto cannabis-tabaco en el embarazo, así como de los efectos asociados con diferentes formas de consumir el cannabis y/o tabaco, si se tiene en cuenta los datos aportados por Holland et al. (2016).

Para Chabarria et al. (2016) resulta necesario informar a las embarazadas sobre los riesgos en el embarazo, más si cabe cuando un $70 \%$ de gestantes y no gestantes consideran leve o nulo el daño que produce el consumo de cannabis una o dos veces por semana (Ko et al., 2015), siendo considerado el cannabis como una droga blanda (Pearson y Shiner, 2002). Esto implica no solo diseñar estudios acerca de la percepción del riesgo que tienen las embarazadas (Moore et al., 2010), más si cabe cuando se ha producido una despenalización de esta droga en algunos países y existe una mayor aceptabilidad de su uso, si no también elaborar protocolos de detección y seguimiento y campañas preventivas desde diferentes ámbitos, no solo el sanitario, dirigidos a los colectivos más vulnerables y con un mayor número de factores de riesgo psicosociales.

Por otro lado, resulta necesario diseñar mecanismos de detección precoz del consumo conjunto en gestantes (Coleman-Cowger et al., 2018) e identificar de forma precoz a los recién nacidos (Stroud et al., 2018), diseñando protocolos de seguimiento y comunicación entre diferentes profesionales sanitarios. Para ello se requiere de instrumentos de evaluación y de screening que aborde el consumo conjunto cannabis-tabaco, frente a instrumentos que emplean el abordaje del consumo de sustancias por separado. Para Holland et al. (2016) se han de incluir en las primeras entrevistas a las embarazadas preguntas acerca del consumo dual.

Por otro lado, dada la relación que parece existir entre el consumo de cannabis antes y durante el embarazo con la presencia o riesgo de manifestar un trastorno por dependencia 
al cannabis (Oga et al., 2017; Peters et al., 2012), se requiere del diseño de intervenciones dirigidas a la deshabituación en embarazadas con consumo dual, tal y como recoge Emery et al. (2016), Rabin y George (2015), SamuelCubizolles et al. (2014) y Stroud et al. (2018). Estas actuaciones han de tener en cuenta las diferentes formas de consumo del cannabis y del tabaco (Coleman-Cowger et al., 2017), así como la frecuencia. Rabin y George (2015) sugieren mayores beneficios psicológicos y neurológicos abordando el abandono de ambas sustancias de forma simultánea.

En base a la evidencia científica, aunque con ciertas limitaciones, se puede concluir que existen efectos negativos del consumo dual cannabis-tabaco durante la gestación sobre el desarrollo fetal, sobre la mujer gestante, en la interacción madre-hijo y en el desarrollo posterior del recién nacido, incluso en la infancia y en la adolescencia.

- Conflicto de intereses.

Los autores declaran no tener ningún conflicto de intereses.

\section{REFERENCIAS}

*Brown, S. J., Mensah, F. K., Ah Kit J., Stuart-Butler, D., Glover, K., Leane, C., ... Yelland, J. (2016). Use of cannabis during pregnancy and birth outcomes in an Aboriginal birth cohort: A crosssectional, population-based study. BMJ Open, 6, e010286. http://dx.doi.org/10.1136/ bmiopen-2015-010286

*Chabarria, K. C., Racusin, D. A., Antony, K. M., Kahr, M., Suter, M. A., Mastrobattista, J. M., y Aagaard, K. M. (2016). Marijuana use and its effects in pregnancy. American Journal of Obstetrics \& Gynecology, 215, 506e, 1-7. http://dx.doi.org/10.1016/i. ajog.2016.05.044

*Coleman-Cowger, V. H., Oga, E. A., Peters, E. N., y Mark, K. (2018). Prevalence and associated birth outcomes of co-use of cannabis and tobacco cigarettes during pregnancy. Neurotoxicology and Teratology, 68, 84-90. https://doi.org/10.1016/i.ntt.2018.06.001

*Coleman-Cowger, V. H., Schaver, G. L., y Peters, E. N. (2017). Marijuana and tobacco co-use among a nationally representative sample of US pregnant and non-pregnant women: 2005-2014 National survey on drug use and health findings. Drug and Alcohol Dependence, 177, 130-135. https://doi.org/10.1016/i. drugalcolep.2017.03.025

*De Genna, N. M., Goldschmidt, L., Richardson, G. A., Cornelius, M. D., y Day, N. L. (2018). Trajectories of pre-and postnatal co-use of cannabis and tobacco predict co-use and drug use disorders in adult offspring. Neurotoxicology and Teratology, 70, 10-17. https://doi. org/10.1016/i.ntt.2018.09.002

*Eiden, R. D., Schuetze, P., Shisler, S., y Huestis, M. A. (2018). Prenatal exposure to tobacco and cannabis: Effects on automatic and emotion regulation. Neurotoxicology and Teratology, 68, 47-54. https://doi.org/10.1016/i. ntt.2018.04.007

*Eiden, R. D.,Zhao, J., Casey,M., Shisler, S.,Schuetze, P., y Colder, C. R. (2018). Pre- and postnatal tobacco and cannabis exposure and child behavior problems: Bidirectional associations, joint effects, and sex differences. Drug and Alcohol Dependence, 185, 82-92. https://doi. org/10.1016/i.drugalcdep.2017.11.038

El Marroun, H. Tiemeier, H., Jaddoe, V. W. V., Hofman, A., Mackenbach, J. P., Steegers, E. A., ... Huizink, A. C. (2008). Demographic, emotional and social determinants of cannabis use in early pregnancy: The generation R study. Drug and Alcohol Dependence, 98(3), 218-226. https:// doi.org/10.1016/i.drugalcdep.2008.05.010.

El Marroun, H., Tiemeier, H., Steegers, E. A., Jaddoe, V. W., Hofman, A., Verhulst, F. C., ... Huizink, A. C. (2009). Intrauterine cannabis exposure affects fetal growth trajectories: the Generation R Study. Journal of the American Academy of Child \& Adolescent, 48(12), 1173-1181. https://doi. org/10.1097/CHl.0b013e3181 bfa8ee.

Emery, R. I., Gregory, M. P., Grace, J. L., y Levine, M. D. (2016). Prevalence and correlates of a lifetime cannabis use disorder among pregnant former tobacco smokers. Addictive Behaviors, 54, 52-58. https://doi.org/10.1016/i. addbeh.2015.12.008

Fariña, F., Arce, R., y Novo, M. (2002). Heurístico de anclaje en las decisiones judiciales [Anchorage 
in judicial decision making]. Psicothema, 14(1), 39-46. Recuperado de http://www.psicothema. com/pdf/684.pdf

Fried, P. A., Watkinson, B., y Willan, A. (1984). Marijuana use during pregnancy and decreased length of gestation. American Journal of Obstetrics and Gynecology, 150(1), 23-27. https://doi.org/10.1016/s00029378(84)80103-9

*Godleski, S. A., Shisler, S., Eiden, R. D., y Huestis, M. A. (2018). Co-use of tobacco and marijuana during pregnancy: Pathways to externalizing behavior problems in early childhood. Neurotoxicology and Teratology, 69, 39-48. https://doi.org/10.1016/i.ntt.2018.07.003

*Gould, G. S., Zeev, Y. B., Tywman, L., Oldmeadow, C., Chiu, S., Clarke, M., y Bonevski, B. (2017). Do clinicians ask pregnant women about exposures to tobacco and cannabis smoking, second-hand-smoke and e-cigarettes? An Australian National Cross-Sectional Survey. International Journal of Environmental Research and Public Health, 14(12):e1585. https://doi. org/10.3390/ijerph 14121585

*Gray, T. R., Eiden, R. D., Leonard, K. E., Conors, G. J., Shisles, S., y Huestis M. A. (2010). Identifying prenatal cannabis exposure and effects of concurrent tobacco exposure on neonatal growth. Clinical Chemistry, 56(9), 1442-1450. https://doi. org/10.1373\%2Fclinchem.2010.147876

Holland, C. I., Rubio, D., Rodriguez, K. L., Kraemer, K. L., Day, N., Arnold, R. M., ... Chang, J. C. (2016). Obstetric health care providers' counseling, responses to pregnant patient disclosures of marijuana use. Obstetrics \& Gynecology, 127(4), 681-687. https://doi. org/10.1097/AOG.0000000000001343

Ko. J. Y., Farr, S. L., Tong, V. T., Greanga, A. A., y Callaghan, W. M. (2015). Prevalence and patterns of marijuana use among pregnant and nonpregnant women of reproductive age. American Journal of Obstetrics and Gynecology, 213, 201.e10. https://doi.org/10.1016/i. ajog.2015.03.021

*Leemaqz, S. Y., Dekker, G. A., McCowan, L. M., Kenny, L. C., Myers, J. E., Simpson, N. A. B., ... SCOPE Consortium (2016). Maternal marijuana use has independent effects on risk for spontaneous preterm birth but not other common late pregnancy complications. Reproductive Toxicology, 62, 77-86. http:// dx.doi.org/10.1016/i.reprotox.2016.04.021

Lester, B. M., ElSohly, M., Wright, L. L., Smeriglio, V. L., Verter, J., Baver, C. R., ... Maza, P. L. (2001). The maternal lifestyle study: Drug use by meconium toxicology and maternal selfreport. Pediatrics, 107(2), 309-317. https:// doi.org/10.1159/000207491.

Liberati, A., Altman, D. G., Tetzlaff, J., Mulrow, C., Gotzsche, P. C., loannidis, J. P., ... Moher, D. (2009). The PRISMA statement for reporting systematic reviews and meta-analyses of studies that evaluate health care interventions: Explanation and elaboration. PLOS Medicine, 6(7), e1000100. https://doi.org/10.1371/ journal.pmed. 1000100

Mark, K., Desai, A., y Terplan, M. (2016). Marijuana use and pregnancy: prevalence, associated characteristics, and birth outcomes. Archives of Women's Mental Health, 19(1), 105-111. https://doi.org/10.1007/s00737. 015-0529-9.

*Massey, S. H., Mroczek, D. K., Reiss, D., Miller, E. S., Jakubowski, J. A., Graham, E. K., ... Shaw, D. S. (2018). Additive drug-specific and sex-specific risks associated with co-use of marijuana and tobacco during pregnancy: Evidence from 3 recent developmental cohorts (2003-2015). Neurotoxicology and Teratology, 68, 97-106. https://doi.org/10.1016/i. ntt.2018.06.002

*Molnar, D. S., Douglas A, Granger, D. A., Shisler, S., y Eiden, R. D. (2018). Prenatal and postnatal cigarette and cannabis exposure: Effects on secretory Immunoglobulin A in early childhood. Neurotoxicology and Teratology, 67, 31-36. https://doi.org/10.1016/i.ntt.2018.03.003.

Moore, D. G., Turner, J. D., Parrott, A. C., Goodwin, J. E., Fulton, S. E., Min, M. O., ... Singer, L. S. (2010). During pregnancy, recreational drug-using women stop taking ecstasy (3, 4 -methylenedioxy- $\mathrm{N}$-methylamphetamine) and reduce alcohol consumption, but continue to smoke tobacco and cannabis: Initial findings from the development and 
infancy study. Journal of Psychopharmacology, 24(9), 1403-1410. https://doi. $\mathrm{org} / 10.1177 \% 2 F 0269881109348165$.

Oga, E. A., Mark, K., y Coleman-Cowger, V. H. (2018). Cigarette smoking status and substance use in pregnancy. Maternal and Child Health Journal, 22(10), 1477-1483. https://doi. org/10.1007/s10995-018-2543-9

Passey, M. E., Sanson-Fisher, R. W., D'Este, C. A., y Stirling, J. M. (2014). Tobacco, alcohol and cannabis use during pregnancy: Clustering of risks. Drug and Alcohol Dependence, 134, 44-50. https://doi.org/10.1016/i. drugalcolep.2013.09.008.

Pearson, G., y Shiner, M. (2002). Rethinking the generation gap: attitudes to illicit drug among young people and adults. Criminology and Criminal Justice, 2(1), 71-86. https://doi.org/ 10.1177/17488958020020010401

Peters, E. N., Budney, A. J., y Carroll, K. M. (2012). Clinical correlates of co-occurring cannabis and tobacco use: A systematic review. Addiction, 107(8), 1404-1417. https://doi.org/10.1111/ j.1360-0443.2012.03843.x

Ogrinc, G., Davies, L., Goodman, D., Batalden, P., Davidoff, F., y Stevens, D. (2015). SQUIRE 2.0 (Standards for Quality Improvement Reporting Excellence): Revised publication guidelines from a detailed consensus process. The Journal of Continuing Education in Nursing, 46(11), 501 507. http://dx.doi.org/10.3928/0022012420151020-02

Rabin, R. A., y George, T. P. (2015). A review of comorbid tobacco and cannabis use disorders: Possible mechanisms to explain high rates of co-use. The American Journal on Addictions, 24(2), 105-116. https://doi.org/10.1111/ ajad. 12186

Ramo, D. E., Liu, H., y Prochaska, J. J. (2012). Tobacco and marijuana use among adolescents and young adults: A systematic review of their co-use. Clinical Psychology Review, 32(2), 105-121. https://doi.org/10.1016/i. cpr.2011.12.002.

Ramo, D. E., Liu, H., y Prochaska, J. J. (2013). Validity and reliability of the nicotine and marijuana interaction expectancy (NAMIE) questionnaire. Drug and Alcohol
Dependence, 137(1-2), 166-170. https://doi. org/10.1016/i.drugalcdep.2012.12.018

Richter, K. P., Kaur, H., Resnicow, K., Nazir, N., Mosier, M. C., y Ahluwalia, J. S. (2004). Cigarette smoking among marijuana users in the United States. Substance Abuse: Official publication of the Association for Medical Education and Research in substance Abuse, 25(2), 35-43.

*Saurel-Cubizolles, M. J., Prunet, C., y Blondel, B. (2014). Cannabis use during pregnancy in France in 2010. BJOG: An International Journal of Obstetrics \& Gynaecology, 121 (8), 971-977. https://doi.org/10.1111/14710528.12626.

Schaver, G. L., Berg, C. J., Kegler, M. C., Donovan, D. M., y Windle, M. (2015). Assessing the overlap between tobacco and marijuana: Trends in patterns of co-use of tobacco and marijuana in adults from 2003-2012. Addictive Behaviors, 49, 26-32. https://doi. org/10.1016/i.addbeh.2015.05.012.

Schaver, G. L., Berg, C. J., Kegler, M. C., Donovan, D. M., y Windle, M. (2016). Differences in tobacco product use among past month adult marijuana users and nonusers: Findings from the 2003-2012 national survey on drug use and health. Nicotine \& Tobacco Research, 18(3), 281-288. https://doi.org/10.1093/ntr/ ntv093

*Schuetze, P., Eiden, R. D., Colder, C. R., Huestis, M. A., y Leonard, K. E. (2018). Prenatal risk and infant regulation: Indirect pathways via fetal growth and maternal prenatal stress and anger. Child Development, 89(2), 123-137. https://doi.org/10.1111/cdev. 12801

*Stroud, L. R., Papandonatos, G. D., McCallum, M., Kehoe, T., Salisbury, A. L., y Huestis, M. A. (2018). Prenatal tobacco and marijuana co-use: Impact on newborn neurobehavior. Neurotoxicology and Teratology, 70, 28-39. https://doi.org/10.1016/i.ntt.2018.09.003

Tinuoye, O., Pell, J. P., y Mackay, D. F. (2013). Meta-analysis of the association between secondhand smoke exposure and physiciandiagnosed childhood asthma. Nicotine \& Tobacco Research, 15(9), 1475-1483. https:// doi.org/10.1093/ntr/ntt033 
Tversky, A. (1977). Features of similarity. Psychological Review, 84(4), 327-352. https://psycnet.apa.org/doi/10.1037/0033295X.84.4.327

Wang, Z., May, S. M., Charoenlap, S., Pyle, R., Ott, N. L., Mohammed, K., y Joshi, A. Y. (2015). Effects of secondhand smoke exposure on asthma morbidity and health care utilization in children: a systematic review and meta-analysis. Annals of Allergy, Asthma \& Immunology, 1 15(5), 396-401. https://doi.org/10.1016/i. anai.2015.08.005

Young-Wolff, K. C., Tucker, L. Y., Alexeeff, S., Armstrong, M. A., Conway, A., Weisner, C., y Goler, N. (2017). Trends in self-reported and biochemically tested marijuana use among pregnant females in California from 20092016. JAMA, 318(24), 2490-2491. https:// doi.org/10.1001/jama.2017.17225 